\title{
A Simple and Effective Technique for Production of Pycnidia and Pycnidiospores by Macrophomina phaseolina
}

\author{
Xinbei Zhao, ${ }^{1}$ Yunxia Ni, ${ }^{1}$ Xintao Liu, ${ }^{1}$ Hui Zhao, ${ }^{1}$ Jing Wang, ${ }^{1}$ Yung-Chun Chen, ${ }^{2}$ Weidong Chen, ${ }^{2}$ and Hongyan Liu ${ }^{1, \dagger}$ \\ ${ }^{1}$ Institute of Plant Protection, Henan Academy of Agricultural Sciences, Key Laboratory of Integrated Pest Management on Crops \\ in Southern Region of North China, Ministry of Agriculture, Henan Key Laboratory of Crops Pest Control, Zhengzhou, Henan, \\ China 450002 \\ ${ }^{2}$ United States Department of Agriculture-Agricultural Research Service, Washington State University, Pullman, WA 99164, \\ U.S.A.
}

\begin{abstract}
Production of pycnidia and pycnidiospores by Macrophomina phaseolina is not often seen in vitro. The objective of this study is to develop a simple and effective technique to obtain pycnidiospores of $M$. phaseolina isolates in vitro and to evaluate germination rates and pathogenicity of pycnidiospores. We found M. phaseolina isolates can produce pycnidia on oatmeal agar (OMA) under ultraviolet light with $365 \mathrm{~nm}$ wavelength (UV). For evaluating the effect of OMA and UV on growth of M. phaseolina, combinations of two agar media and three lighting

90\%. In pathogenicity tests, inoculation with the pycnidiospores showed symptoms later than inoculation with hypha-colonized toothpicks. Significant differences in the pathogenicity is detected between isolates Mp2014003 and Mp2014024 when inoculation is done with the pycnidiospores $(P<0.001)$, but not when hypha-colonized toothpicks are used as inoculum $(P=0.091)$. This study provides a new method for obtaining pycnidiospores of $M$. phaseolina for future investigations.
\end{abstract} conditions were tested. The results confirm that all six M. phaseolina isolates produced pycnidia only on OMA under UV. The pycnidiospores produced on OMA under UV had germination rates higher than
Keywords: Macrophomina phaseolina, oatmeal agar, ultraviolet light, sporulation, pathogenicity
Macrophomina phaseolina (Tassi) Goid., one of the most important soil-borne pathogens of sesame, causes stem blight of sesame resulting in significant economic damage and nutritional loss. M. phaseolina is widespread in the warm climate regions, causing charcoal rot in the roots and lower stems of at least 850 hosts (Dhingra and Sinclair 1978; Farr and Rossman 2019). Though pycnidia are commonly found on many host plants including sesame (Hildebrand et al. 1945; Kulkarni and Patil 1968; Kulkarni et al. 1962; Mathur 1967; Moniz et al. 1956), the fungus only produces microsclerotia on the common medium PDA in culture. The production of pycnidia by $M$. phaseolina can be induced on more complex media that contain different plant tissues, seed, or plant extracts (Chidambaram and Mathur 1975; Crous et al. 2006; Goth and Ostazeski 1965; Knox-Davies 1965, 1966; Ma et al. 2010; Machado 1987; Oosthuizen and Potgieter 1974; Premamalini et al. 2012). However, preparation of these media is complicated or time-consuming, or the production of pycnidia may not be reproducible when we attempted to induce pycnidia production of the isolates from sesame in China according to the methods of Premamalini et al. (2012) and Crous et al. (2006).

A number of fungal pathogens readily produce spores on oatmeal agar (OMA). For example, OMA and potato sucrose agar are the best for spore production and growth of Ampelomyces quisqualis, a hyperparasitic fungus of powdery mildew fungi (Liang et al. 2004). Similarly, OMA is the suitable medium for sporulation by Alternaria alternata causing tobacco brown spot disease and by Fusarium acuminatum causing Fusarium fruit rot (Wang et al. 2017; Yang et al. 2012). In addition, Phytophthora erythroseptica produces oospores in OMA at $20^{\circ} \mathrm{C}$ for $4-6$ weeks (Gregg 1957). Interestingly, Zhang et al. (2015) found that Didymella bryoniae produced greater

${ }^{\dagger}$ Corresponding author: H. Y. Liu; liuhy1219@163.com

Funding: This work was financially supported by the earmarked fund for China Agriculture Research System (CARS-14).

The author(s) declare no conflict of interest.

Accepted for publication 29 November 2019.

C 2020 The American Phytopathological Society numbers of pycnidia when cultures were placed under ultraviolet light, although the isolates produced few pycnidia on OMA under regular lighting conditions. Also, two isolates of M. phaseolina from okra (Hibiscus esculentus) and bean (Phaseolus vulgaris) formed more pycnidia under ultraviolet light than under artificial daylight on water-agar-leaf medium (Chidambaram and Mathur 1975). Similarly, ultraviolet radiation was often used to induce production of pycnidia by M. phaseolina (Chidambaram and Mathur 1975; Crous et al. 2006; Goth and Ostazeski 1965; Knox-Davies 1965, 1966; Ma et al. 2010; Machado 1987; Oosthuizen and Potgieter 1974).

According to the aforementioned studies, we selected OMA and ultraviolet light with $365 \mathrm{~nm}$ wavelength (UV) to induce pycnidia in $M$. phaseolina isolated from sesame. Ultraviolet light with $365 \mathrm{~nm}$ wavelength can penetrate the glass of a petri dish to affect the growth of M. phaseolina isolates (Pinnell and Madey 1998; Tuchinda et al. 2006). For evaluating the effect of OMA and UV on growth of $M$. phaseolina, diameters of the colonies and sclerotia were measured on two media (OMA and PDA) with three different lighting conditions including full dark, $16 \mathrm{~h}$ white light and $8 \mathrm{~h}$ dark, and UV. The germination rates and pathogenicity of the pycnidiospores were determined to evaluate the vitality and availability of pycnidiospores produced on OMA under UV.

\section{Materials and Methods}

Media and lighting conditions. Two agar media were tested in this study. Potato dextrose agar (PDA, pH 6.4) consisted of $200 \mathrm{~g}$ potato boiled in $1,000 \mathrm{ml}$ distilled water, 15 to $20 \mathrm{~g}$ agar, $20 \mathrm{~g}$ dextrose, and distilled water to a final volume of 1,000 ml. PDA without agar was PDB. OMA consisted of $60 \mathrm{~g}$ oatmeal and $15 \mathrm{~g}$ agar. Oatmeal was passed through a 60 -mesh sieve, and then boiled in $1,000 \mathrm{ml}$ distilled water. The $\mathrm{pH}$ of OMA was adjusted to 6.8. The media were autoclaved for $15 \mathrm{~min}$ at $121^{\circ} \mathrm{C}$.

The cultures were exposed to three lighting conditions: full dark; $16 \mathrm{~h}$ white light and $8 \mathrm{~h}$ dark; ultraviolet light. White light was $100 \%$ illumination of FPG3 three-temperature zone light incubator (Ningbo Life Technology Co., LTD, Zhejiang, China). A black light lamp was used to provide ultraviolet light. The black light lamp has a wavelength of $365 \mathrm{~nm}$ and a power of $18 \mathrm{~W}$, which consists of two U-tubes (Cnlight Electro-Optic Co., Ltd., Guangdong, China). 
M. phaseolina isolates. Only two of 208 isolates collected from sesame produced pycnidia on PDA in $16 \mathrm{~h}$ white light and $8 \mathrm{~h}$ dark, so we have been looking for new ways to induce pycnidia production by $M$. phaseolina in vitro. In a preliminary study for evaluating sporulation of M. phaseolina on OMA under UV, 23 isolates of M. phaseolina were first arbitrarily selected from the $206 \mathrm{M}$. phaseolina isolates that did not produce pycnidia on PDA in $16 \mathrm{~h}$ white light and $8 \mathrm{~h}$ dark. We found 15 of these 23 selected isolates could produce pycnidia on OMA under UV. Six of the 15 isolates that did form pycnidiospores were arbitrarily selected for evaluating production and germination rates. These six isolates of $M$. phaseolina, Mp2010010, Mp2013058, Mp2014003, Mp2014023, Mp2014024, and Mp2014141, were collected from different sesame production areas in China (Table 1). These isolates were preserved in key laboratory of integrated pest management on crops in southern region of North China, Ministry of Agriculture, Henan key laboratory of crops pest control of the Plant Protection Institute, Henan Academy of Agricultural Sciences.

Effect of media and light treatment on sporulation. The $M$. phaseolina isolates were grown on PDA plates at $28^{\circ} \mathrm{C}$ under $16 \mathrm{~h}$ white light and $8 \mathrm{~h}$ dark for 4 days. Agar blocks ( $5 \mathrm{~mm}$ diameter) were cut with a sterile borer from the colony margin on PDA cultures and transferred to the center of PDA and OMA plates overlaid with a sterile cellophane (Solarbio Science \& Technology Co., Ltd., Beijing, China) membrane ( $75 \mathrm{~mm}$ diameter) on the agar surface. The PDA and OMA plates were incubated initially in the dark at $28^{\circ} \mathrm{C}$ for $24 \mathrm{~h}$, and then were incubated at three light treatments: 1) dark, 2) $16 \mathrm{~h}$ white light and $8 \mathrm{~h}$ dark, and 3) $\mathrm{UV}$ at $28^{\circ} \mathrm{C}$ for $120 \mathrm{~h}$, which is a total of $144 \mathrm{~h}$ after transferring. There were three replicates for each isolate in each medium-light combination. All plates were arranged randomly in a single layer. Production of pycnidia was inspected every $24 \mathrm{~h}$ with the aid of a COCI BA3000i biomicroscope at $\times 200$ magnification, and colony diameters of the mycelium and the diameters covered with sclerotia were measured at $48 \mathrm{~h}$ after transferring.

Quantification of pycnidia production. In order to remove the interference of hyphae on counting pycnidia, $75 \%$ ethanol was sprayed on the surface of OMA plates (Ma et al. 2010). Pycnidia in each OMA plate were visually counted with the aid of a Leica inverted stereo microscope at $\times 10$ magnification. Four microscopic fields were randomly chosen in every plate, with three plates per isolate. Each plate was the experimental unit for statistical analysis.

Quantification of pycnidiospore production. Pycnidia and pycnidiospores from each isolate were collected by scraping the pycnidia on the cellophane with a sterile scalpel blade in each replicate, three replicates of each isolate, followed by rinsing with $5 \mathrm{ml}$ sterile $0.05 \%$ Tween-20 solution to remove the pycnidia and pycnidiospores from the plates. Then, the rinsing fluid of $0.05 \%$ Tween- 20 solution was collected into $10-\mathrm{ml}$ centrifuge tubes, followed by shock on a vortex mixer for 20 min until the pycnidia were broken to release pycnidiospores. The pycnidiospore suspensions were filtered through a sterile $37-\mu \mathrm{m}$ nylon mesh to remove debris and hypha fragments. The

Table 1. List of Macrophomina phaseolina isolates used in study

\begin{tabular}{llc}
\hline Isolate & Year & \multicolumn{1}{c}{ Collecting locations } \\
\hline Mp2010010 & 2010 & $\begin{array}{c}\text { Longquan Village, Heqiao Town, } \\
\text { Xuyi County, Jiangsu Province } \\
\text { The Test Base of Henan Academy of } \\
\text { Agricultural Sciences in Yuanyang } \\
\text { City } \\
\text { Gaoshi Township, Xiangcheng City, } \\
\text { Zhoukou City, Henan Province }\end{array}$ \\
Mp2014003 & 2013 & $\begin{array}{c}\text { Experimental Station in Luohe City, } \\
\text { Henan Province }\end{array}$ \\
Mp2014023 & 2014 & $\begin{array}{c}\text { Experimental Station in Luohe City, } \\
\text { Henan Province } \\
\text { Chitang Village, Meilin Town, } \\
\text { Fengcheng City, Jiangxi Province }\end{array}$ \\
\hline
\end{tabular}

number of pycnidiospores in each plate was estimated with the aid of a hemocytometer.

Germination of pycnidiospores. M. phaseolina isolates were cultured on OMA under UV for $120 \mathrm{~h}$. Mature pycnidia were placed on the glass slide with one drop of $0.05 \%$ Tween- 20 solution, which was covered with a coverslip. Pycnidiospores were released by gently pressing the coverslip against the pycnidia. The water containing the pycnidiospores was collected with a micropipette and transferred into a $1.5-\mathrm{ml}$ centrifuge tube. Water agar $(2 \% \mathrm{wt} / \mathrm{vol})$-coated slides were placed in a tray with four layers of sterile gauze soaked with sterile water. Fifteen microliters of the pycnidiospore suspensions were evenly spread on water agar slides, and each isolate repeated three times. The tray covered with preservative film was placed in an incubator at $28^{\circ} \mathrm{C}$. Pycnidiospores with germ tubes longer than half of the spore width were considered to be germinated. Percentages of germination of 200 pycnidiospores were determined after incubation of 8 and $24 \mathrm{~h}$ at $\times 200$ magnification under a COCI BA3000i biomicroscope.

Pathogenicity test. For evaluating the pathogenicity of pycnidiospores, the three isolates with higher germination rates of pycnidiospore, Mp2014003, Mp2014024, and Mp2014141, were tested on sesame stems by adapting the method of Yildiz and Benlioğlu (2014). Sesame stems (10 cm in length) of the susceptible variety '9014' growing on seedling-raising pots were collected from healthy plants at the six-leaf stage. The stems were disinfected with $75 \%$ ethanol for $5 \mathrm{~min}$, followed by washing three times with sterile water, and then air-dried on sterile tissue papers. Two pieces of sterile tissue papers saturated with sterile distilled water were placed in sterile stainless-steel trays. The stems were slightly wounded at the middle with a toothpick before inoculation. The inoculation was carried out in two ways: by placing a hypha-colonized toothpick ( $1 \mathrm{~cm}$ in length) and by placing $10 \mu \mathrm{l}$ pycnidiospore suspension $\left(5 \times 10^{4}\right.$ pycnidiospores/ $\mathrm{ml}$ ) on the wound. To prepare the hypha-colonized toothpicks, toothpicks were first boiled in PDB for $1 \mathrm{~h}$, and autoclaved for $15 \mathrm{~min}$ at $121^{\circ} \mathrm{C}$. Then, the sterile toothpicks were placed in PDA plate covered with M. phaseolina colonies and incubated in the dark at $28^{\circ} \mathrm{C}$ for 3 days. Control stems of the two inoculation methods were inoculated with only a sterile toothpick or sterile water, respectively. There were five sesame stems for each treatment. The stainless-steel trays were wrapped with preservative film to prevent moisture loss and incubated at $28^{\circ} \mathrm{C}$. Development of symptoms was observed every $12 \mathrm{~h}$ after inoculation. The lesion length was measured at 3 days after inoculation.

Statistical analysis. All experiments were conducted three times. Statistical analyses were performed using SPSS v17.0 software (SPSS, Chicago, IL, U.S.A.). Comparisons of six isolates for diameter of mycelium and sclerotium, germination rate of pycnidiospores and three isolates for lesion length were made by Duncan's method at a significance level of $P=0.05$. In order to correct for unequal variance, the number of pycnidia and pycnidiospores was transformed by taking the logarithm to the base 10 prior to analysis of variance.

\section{Results}

Effect of media and light treatment on sporulation. All six isolates produced pycnidia only on OMA under UV light at $72 \mathrm{~h}$; pycnidia were not observed on PDA either under UV light or under $16 \mathrm{~h}$ white light and $8 \mathrm{~h}$ dark up to $144 \mathrm{~h}$ after transferring. Under the condition of $16 \mathrm{~h}$ white light and $8 \mathrm{~h}$ dark, only one isolate (Mp2014024) produced 2 to 3 pycnidia per plate up to $96 \mathrm{~h}$ on OMA. In full dark, none of the isolates produced pycnidia either on OMA or on PDA. All six M. phaseolina isolates readily produced sclerotia under all tested conditions.

For mycelial growth and sclerotial formation, mean colony diameters of mycelium under UV were significantly larger than in full dark or $16 \mathrm{~h}$ white light and $8 \mathrm{~h}$ dark conditions (Fig. 1A). Mean diameters covered with sclerotia on OMA were significantly smaller than on PDA (Fig. 1B).

Number of pycnidia and pycnidiospores per plate. The number of pycnidia and pycnidiospores differed significantly among the six M. phaseolina isolates. Isolate Mp2014023 produced the greatest number of pycnidia $\left(1.5 \times 10^{4}\right.$ pycnidia per plate $)$ and pycnidiospores 
$\left(3.3 \times 10^{6}\right.$ pycnidiospores per plate) on OMA (Fig. 2), while Mp2014141 produced the least number of pycnidia $\left(4.8 \times 10^{3}\right.$ pycnidia/plate) and pycnidiospores $\left(8.9 \times 10^{5}\right.$ pycnidiospores per plate). Pycnidiospores produced by other isolates were above $1.4 \times$ $10^{6}$ pycnidiospores per plate except for isolate Mp2014141.

The germination of pycnidiospores. The pycnidiospores produced by the $M$. phaseolina isolates on OMA under UV had excellent germination ability on water agar slides (Fig. 3). The germination rates were over $88 \%$ and $93 \%$ after incubation 8 and $24 \mathrm{~h}$, respectively. Isolates Mp2014003, Mp2014024, and Mp2014141 had higher germination rates than the other three isolates after incubation for $24 \mathrm{~h}$.

The pathogenicity of pycnidiospores to stem in vitro. The onset of symptoms was $24 \mathrm{~h}$ after inoculation with hypha, while the onset of symptoms was $36 \mathrm{~h}$ after inoculation with pycnidiospore. Typical dark-brown lesions developed on the inoculation sites. No symptoms were observed on the control stems. The lesion sizes varied among the isolates. The lesions of hypha inoculation ranged from 30.2 to $52.2 \mathrm{~mm}$, while the lesions of pycnidiospore inoculation ranged from 3.8 to $35.0 \mathrm{~mm}$ (Fig. 4). Based on lesions size, the infection on sesame stem with hypha of M. phaseolina was faster than with pycnidiospores. The ability of each isolate to cause lesions was similar, from strong to weak: Mp2014003, Mp2014024, and Mp2014141, regardless of pycnidiospores or hypha used as inoculum. The aggressiveness of Mp2014003 was significantly higher than that of Mp2014024 with pycnidiospores as inoculum $(P<0.001)$, but no difference was observed when mycelia were used as inoculum $(P=$ 0.091).

\section{Discussion}

Production of pycnidia and pycnidiospores by M. phaseolina is not common in culture, but pycnidiospores are frequently required for quantitative inoculation studies of pathogenicity of M. phaseolina, screening resistant sesame varieties, and pathogenic mechanisms of pycnidiospores. M. phaseolina was artificially inoculated on hosts to produce pycnidia in earlier studies. Then, various media containing special plant ingredients were investigated for production of pycnidia in vitro. Sterile pine needles were used to induce sporulation in M. phaseolina isolated from dead or dying twigs of woody plants (Crous et al. 2006). Similarly, sterile banana peel culture was used to induce pycnidia and pycnidiospores of $M$. phaseolina isolated from the injured eye of a 70-year-old patient (Premamalini et al. 2012). Furthermore, plant extracts, like peanut butter extract, soynut butter extract, or canola oil, were used to induce pycnidia for M. phaseolina isolates collected from soybean (Ma et al. 2010). In our preliminary study, M. phaseolina isolate Mp2010010 did not produce pycnidia and pycnidiospores based on the methods of Premamalini et al. (2012) and Crous et al. (2006), but this isolate produced pycnidia and pycnidiospores on OMA under UV. It is possible that production of pycnidia and pycnidiospores for different $M$. phaseolina isolates require different nutrition in vitro. So, we provide a new choice for sporulation of $M$. phaseolina in vitro. Genetic differences among the isolates could explain why the other 8 isolates could not produce pycnidia in the initial test of $23 \mathrm{M}$. phaseolina on OMA under UV. The genetic differences of the isolates between sporeproducing and non-spore-producing on OMA under UV should be further investigated.

The production of pycnidia on OMA under UV occurred sooner in this study than in previous studies. Ma et al. (2010) reported that pycnidium first appeared 6 days after inoculation with $1.1 \times 10^{3}$ pycnidia

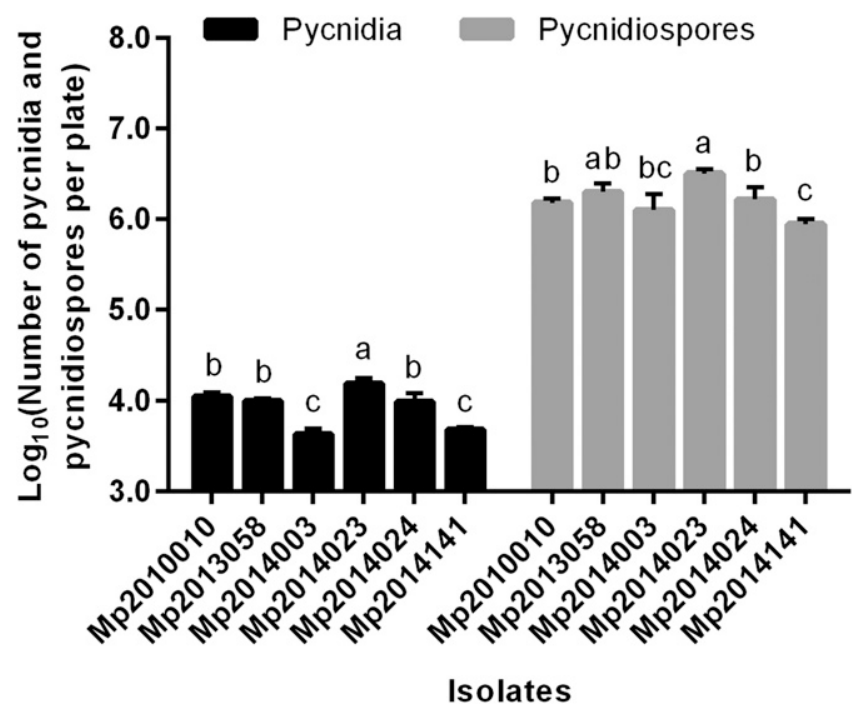

Fig. 2. Production of pycnidia and pycnidiospores per plate produced by six Macrophomina phaseolina isolates on oatmeal agar (OMA). Bars in the same group marked by the same letter do not differ significantly at $P=0.05$.

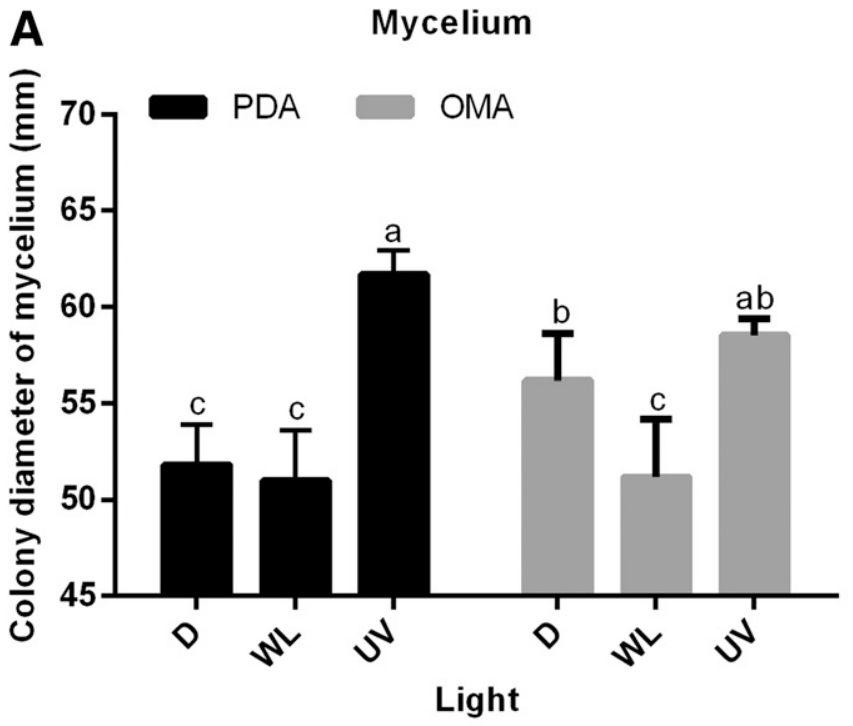

B

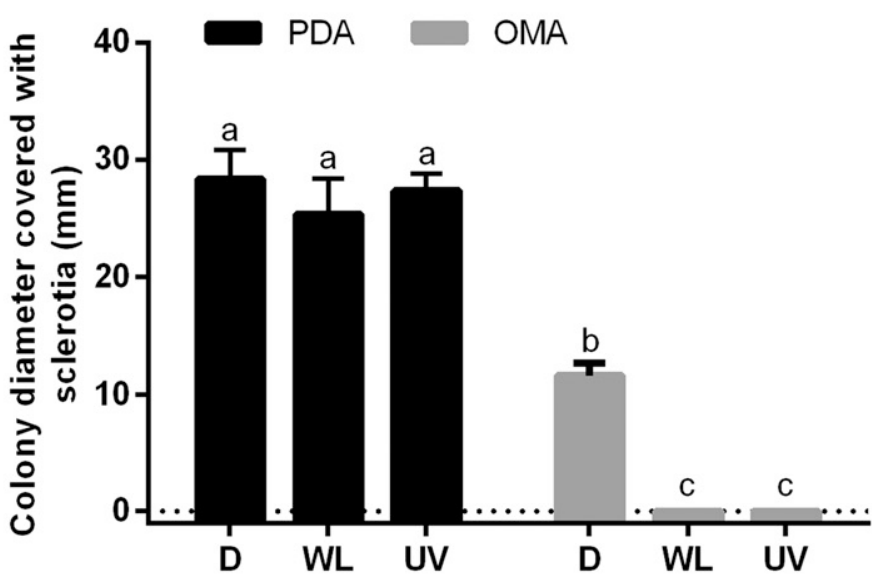

Light

Fig. 1. The mean values $\mathbf{A}$, of colony diameters of mycelium and $\mathbf{B}$, of diameters covered with sclerotia of six Macrophomina phaseolina isolates at $48 \mathrm{~h}$ in different treatments: potato dextrose agar (PDA) and oatmeal agar (OMA) in the dark (D), $16 \mathrm{~h}$ white light and $8 \mathrm{~h}$ dark (WL) and under ultraviolet light (UV). Bars in all columns (either mycelium or sclerotia) marked by the same letter do not differ significantly at $P=0.05$. 
per plate on peanut butter extract-saturated filter paper placed over soynut butter extract agar. In this study, it took only 3 days after inoculation with the highest number at $1.6 \times 10^{4}$ pycnidia per plate. The OMA has clear advantages over other reported media. For example, the ingredients are low cost and easy to conserve and obtain, the process of preparation is simple, less time-consuming, low requirement for laboratory conditions, and there are no inflammable and explosive extracting reagents involved. Based on colony diameters of mycelium and diameters covered with sclerotia in different treatment combinations, the UV promoted mycelium growth, while OMA reduced the area in which sclerotia was formed on the plate.

In previous studies, sclerotia-colonized toothpicks or sclerotia suspension were used as inoculum (Koike 2008; Zveibil and Freeman 2005). Sclerotia on toothpicks or in suspension are difficult to separate with hypha completely, which makes it difficult to standardize the amount of inoculum among experimental runs. A very high

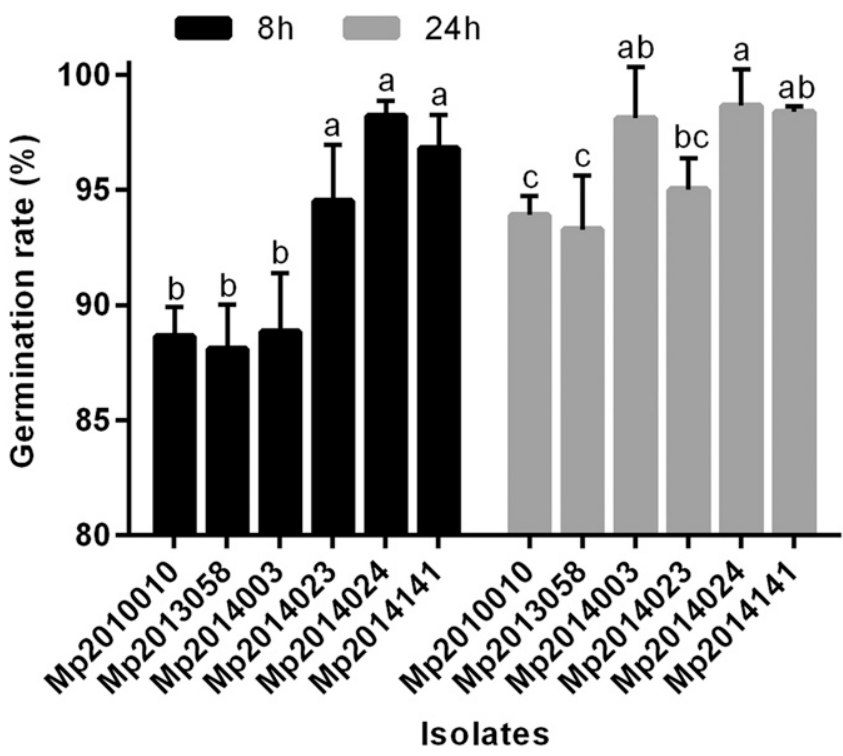

Fig. 3. Percent germination of pycnidiospores of six Macrophomina phaseolina isolates at 8 and $24 \mathrm{~h}$ after incubation. Bars in the same group marked by the same letter do not differ significantly at $P=0.05$.

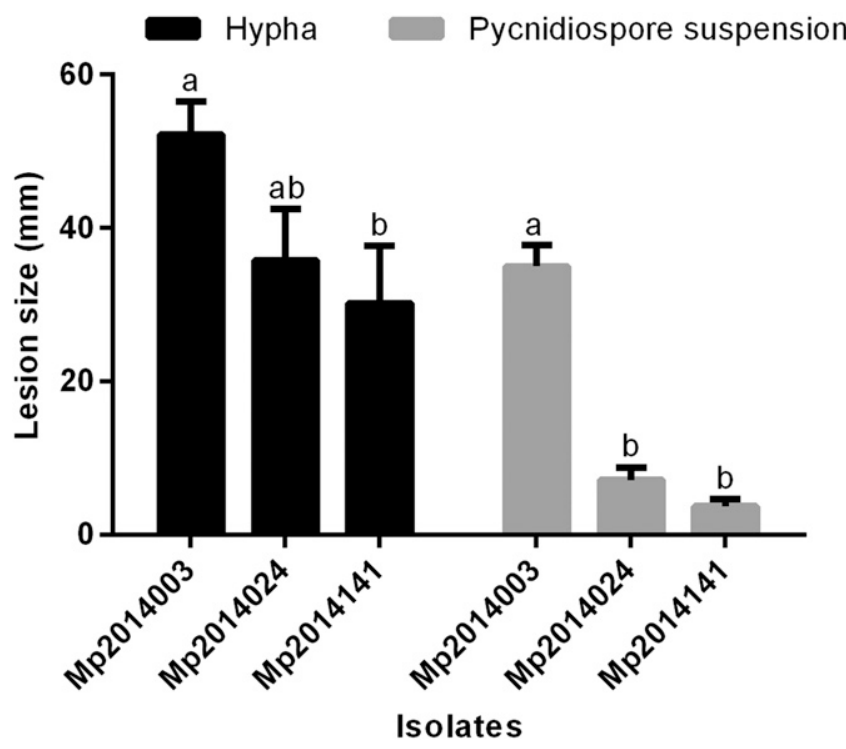

Fig. 4. Lesion sizes caused by three Macrophomina phaseolina isolates on sesame stems when hypha or pycnidiospore was used as inoculum. Bars in the same group marked by the same letter do not differ significantly at $P=0.05$. percentage (above 90\%) of pycnidiospores produced on OMA germinated, suggesting pycnidiospores could serve as inoculum to study the pathogenicity and infection histology of $M$. phaseolina. Consequently, we evaluated pycnidiospores as an inoculum source, since it is easier to quantify the number of pycnidiospores in the inoculum and thus standardize inoculum among treatments. In our pathogenicity tests, symptoms were observed later using the pycnidiospore suspensions than using hypha on toothpicks. That is most likely due to the fact that hypha can expand directly in sesame stem, while pycnidiospores can only expand after germination, and higher biomass in hypha than in the pycnidiospores. Differences in the pathogenicity among the isolates were detected when inoculation was done with pycnidiospores but not with hypha, suggesting that pycnidiospores as inoculum are more sensitive for detecting variation in pathogenicity.

M. phaseolina stem blight is a polycyclic disease, and its pycnidiospores were responsible for the secondary infection of Phaseolus vulgaris (Luttrell and Garren 1952). Obtaining pycnidiospores by this easy and efficient technique with OMA under UV light condition will speed up the research on infection histology and pathogenic mechanism of pycnidiospores, further contributing to the research on secondary infection and epidemic dynamics of stem blight of sesame in the field.

\section{Literature Cited}

Chidambaram, P., and Mathur, S. B. 1975. Production of pycnidia by Macrophomina phaseolina. Trans. Br. Mycol. Soc. 64:165-168, IN11.

Crous, P. W., Slippers, B., Wingfield, M. J., Rheeder, J., Marasas, W. F., Philips, A. J., Alves, A., Burgess, T., Barber, P., and Groenewald, J. Z. 2006. Phylogenetic lineages in the Botryosphaeriaceae. Stud. Mycol. 55:235-253.

Dhingra, O. D., and Sinclair, J. B. 1978. Biology and pathology of Macrophomina phaseolina. Imprensia Universidade Federal de Vicosa, Vicosa, Brazil.

Farr, D. F., and Rossman, A. Y. 2019. Fungal Databases. U.S. National Fungus Collections, ARS, USDA. Retrieved August 21, 2019, from https://nt.arsgrin.gov/fungaldatabases/

Goth, R., and Ostazeski, S. 1965. Sporulation of Macrophomina phaseoli on propylene oxide-sterilized leaf tissues. Phytopathology 55:1156.

Gregg, M. 1957. Germination of oospores of Phytophthora erythroseptica. Nature 180:150.

Hildebrand, A. A., Miller, J. J., and Koch, L. W. 1945. Some studies on Macrophomina phaseoli (Maubl.) Ashby in Ontario. Sci. Agric. 25:690-706.

Knox-Davies, P. S. 1965. Pycnidium production by Macrophomina phaseoli. S Afr. J. Agric. Sci. 8:205-218.

Knox-Davies, P. S. 1966. Further studies on pycnidium production by Macrophomina phaseoli. S. Afr. J. Agric. Sci. 9:595-600.

Koike, S. T. 2008. Crown rot of strawberry caused by Macrophomina phaseolina in California. Plant Dis. 92:1253.

Kulkarni, N. B., and Patil, B. C. 1968. Studies on the pycnidial formation by Macrophomina phaseoli (Maubl.) Ashby size of pycnidia, ostiole and pycnidiospores and its significance. Mycopathol. Mycol. Appl. 36:311-321.

Kulkarni, N. B., Patil, B. C., and Sulaiman, M. 1962. Pycnidial formation by Macrophomina phaseoli on artificially inoculated cotton. Phytopathology 52: 369-372.

Liang, C., Zhao, H. H., Li, B. D., Zhang, Y. C., and Lv, G. Z. 2004. Biological characteristics of Ampelomyces quisqualis hyperparasite on tickseed powdery mildew. Yunnan Nong Ye Da Xue Xue Bao 19:648-652.

Luttrell, E. S., and Garren, K. H. 1952. Blights of snap bean in Georgia. Phytopathology 42:607-613.

Ma, J., Hill, C. B., and Hartman, G. L. 2010. Production of Macrophomina phaseolina conidia by multiple soybean isolates in culture. Plant Dis. 94: 1088-1092.

Machado, C. C. 1987. Macrophomina phaseolina: Biological behavior of isolates, spatial pattern of microsclerotia in the soil, and incidence on soybeans. University of Illinois at Urbana-Champaign.

Mathur, S. B. 1967. Pycnidia formation in Sclerotium bataticola Taub. on sunflower. J. Phytopathol. 58:137-140.

Moniz, L., Thirumalachar, M. J., and Patel, M. K. 1956. Technique for artificial inoculation of cotton plants with Macrophomina phaseoli. Curr. Sci. 25:64.

Oosthuizen, M. M. J., and Potgieter, D. J. J. 1974. Induction of photosporogenesis in Macrophomina phaseoli by an octadecenoic acid from peanuts. Phytochemistry 13:1027-1029.

Pinnell, S. R., and Madey, D. L. 1998. Topical vitamin C in skin care. Aesthet. Surg. J. 18:468-470.

Premamalini, T., Ambujavalli, B. T., Vijayakumar, R., Rajyoganandh, S. V. Kalpana, S., and Kindo, A. J. 2012. Fungal keratitis caused by Macrophomina phaseolina-A case report. Med. Mycol. Case Rep. 1:123-126.

Tuchinda, C., Srivannaboon, S., and Lim, H. W. 2006. Photoprotection by window glass, automobile glass, and sunglasses. J. Am. Acad. Dermatol. $54: 845-854$. 
Wang, C. W., Wang, Y., Zhang, X. Q., Zhang, Z. G., Wang, M. Q., and Wang, J. M. 2017. Determination of biological characteristics and preliminary screening of control fungicides of Fusarium acuminatum causing Fusarium fruit rot on blueberry. Acta Hortic. Sin. 44:15891598.

Yang, L. I. U., Chen, Z. E. N. G., Xiang, H. Q., and Jiang, Y. L. 2012. Study on biological characteristics of Alternaria alternata of Guizhou province. Guangdong Agric. Sci. 9:77-79.
Yildiz, A., and Benlioğlu, S. 2014. A laboratory bioassay for evaluating pathogenicity of Macrophomina phaseolina and Rhizoctonia solani isolates to strawberry stolons. Phytoparasitica 42:367-369.

Zhang, L. J., Zhang, H. L., Ha, K. W., and Wang, J. S. 2015. Isolation and identification of pathogen of Didymella bryoniae and spore production induction of conidium. J. Ningxia University (Natural science edition) 36:382-385.

Zveibil, A., and Freeman, S. 2005. First report of crown and root rot in strawberry caused by Macrophomina phaseolina in Israel. Plant Dis. 89:1014. 